\title{
Qualitative Assessment of Factors Affecting Prevention of Mother-To- Child Transmission (PMTCT) of HIV Programs in University Teaching Hospitals in Anambra State, Nigeria
}

\author{
Article by Dada Michael Olugbamila ${ }^{1}$, Amosu, Ademola $\mathrm{M}^{2}$, Ogbodo, Uchechukwu Chibuzo ${ }^{3}$ \\ ${ }^{1}$ HSS and Lab Department, State Ministry of Health, Nigeria \\ ${ }^{2}$ Department of Public Health, School of Public \& Allied Health, Babcock University, Nigeria \\ ${ }^{3} M \& E$ Department, State Ministry of Health, Nigeria. \\ E-mail: ${ }^{1}$ gbamidada25@yahoo.com
}

\begin{abstract}
Background: Human Immunodeficiency Virus (HIV) infection in newborns is one of the leading causes of infant mortality globally and accounted for about 1.8million childhood deaths in 2010. Despite international interventions aimed at reducing pediatric infections at antenatal clinic entry points, however, there is still limited access and utilization of Prevention of Mother to Child Transmission (PMTCT) services resulting in low target coverage due to obstacles existing at facility, community and state levels. Anambra State still records a low patronage of PMTCT interventions by pregnant mothers.

Objective: This study was carried out to determine the factors affecting prevention of mother-to-child transmission (PMTCT) of HIV programs in university teaching hospitals in Anambra state, Nigeria qualitatively.

Methods: This is a descriptive cross-sectional study involving 128 randomly selected HIV positive pregnant women attending antenatal care clinics and service providers of two purposively selected teaching hospitals in Anambra state, Nigeria. Qualitative data were obtained through focus group discussions and key in-depth informant interviews. Data analysis was carried out using NVivo version 9 qualitative data analysis Software.

Results: The findings of this work show that ninety-nine percent (99\%) of the study participants knew that HIV was a deadly virus transmitted through sex and other blood contact routes. Seventy six percent (76\%) of the participants knew that HIV could be transmitted from mother to child, while about $53 \%$ of them knew the major ways of MTCT of HIV. The participants that did not know how HIV could be passed from mother to child were $41 \%$. Others factors are lack of awareness and knowledge about HCT, late presentation at ANC clinics, low numbers of PMTCT centers within locality of respondents, poor involvement of male partners/spouses and stigma were the main reasons cited for poor utilization of PMTCT programs in the state.

Conclusion: The study highlights a referral linkage of traditional birth attendants to health facilities for PMTCT interventions in the state to have profound impact on the health of the mother, child and nation at large.
\end{abstract}

Keywords: Human immunodeficiency virus (HIV), Prevention of mother to child transmission (PMTCT), HIV counselling and testing, Antenatal Care.

\section{Background}

HIV infection among women of child-bearing age and mother-to-child transmission (MTCT) of HIV in Nigeria remain major health issues (Ezeanolue, et al., 2013; Nkwo, 2013). Worldwide, approximately 39.5 million people are living with HIV/AIDS, including an estimated 17.7 million women and 2.3 million children under the age of 15 . Women currently represent the population which reflects the fastest increase in HIV infection rates; in the hardest hit countries of sub-Saharan Africa, more than $60 \%$ of all new HIV infections are occurring in women, infants, and young children with 1400 children under the age of 15 being infected with HIV every day. In 2005 alone, an estimated 540,000 children were newly infected with HIV, with approximately $90 \%$ of these infections occurring in sub-Saharan Africa. Without appropriate care and treatment, more than $50 \%$ of newly infected children will die before their second year in life (WHO, 2007). In Nigeria in 2009, according to the Nigerian National Agency for the Control of AIDS (NACA), about 1.72 million women and girls were living with HIV and AIDS with the highest prevalence 
Texila International Journal of Public Health

Volume 5, Issue 3, Sep 2017

rate of 5.6\% among women in the age group 25-29 (NACA, 2011). In 2012, there were 110,000 new HIV infections among women aged 15-49 years in Nigeria, ranking the country second (next only to South Africa) among countries with the highest burden of new HIV infections among women (UNAIDS, 2013). Nigeria is one of 22 countries that account for $90 \%$ of pregnant women living with HIV (Ezeanolue et al., 2013). In 2010, despite improved efforts dedicated to the prevention of mother-to-child HIV transmission (PMTCT), only $16.9 \%$ of pregnant women in Nigeria were tested for HIV (NACA, 2012). The low rates of testing and treatment in Nigeria contributed to an estimated 75,000 HIV-infected infants in 2010 (Ezeanolue et al., 2013, WHO, 2011). In 2012, while these rates dropped, 60,000 new HIV infections occurred among children, now making Nigeria the country with the largest number of children acquiring HIV through MTCT (UNAIDS, 2013). Currently, Nigeria alone, accounts for $30 \%$ of the burden of MTCT (UNAIDS, 2011).

Approximately $90 \%$ of HIV infection among children is acquired through mother to child transmission of HIV (MTCT). Transmission from an HIV-positive pregnant woman to her child can occur during pregnancy, labour and delivery, or through breastfeeding (MOHSS, 2008). The risk of MTCT can be reduced to less than $2 \%$ with a package of evidence-based interventions including ARV prophylaxis and treatments combined with elective caesarean section and avoidance of breastfeeding. Infant feeding patterns are a very important determinant of MTCT. For mothers using replacement feeding there is obviously no transmission through breastfeeding (WHO, 2007). Breastfeeding during a period of 6 months leads to approximately $10 \%$ extra transmission (from $20 \%$ to $30 \%$ ), while breastfeeding during a period of 18-24 months leads to approximately $17.5 \%$ extra transmission (from $20 \%$ to $37.5 \%$ ), compared to no breastfeeding (De Cock et al, 2000).

Interventions to reduce pediatric HIV infection have become readily available worldwide, especially in low and middle-income countries. In 2009, 53\% of HIV-infected pregnant women worldwide received antiretroviral (ARV) drugs to prevent mother-to-child transmission (WHO, 2010). While coverage is increasing in sub-Saharan Africa, ranging from $8 \%$ in some settings to 54\% in others (WHO, 2007), PMTCT programs in the continent are still plagued by multiple problems. For instance, many HIV positive pregnant women still face constraints in accessing ARV drugs because they refuse to participate or are lost to follow-up in existing programs. Health system factors (critical shortage of personnel, lack of skilled attendant at birth, poor infrastructure, and inadequate supply of PMTCT kits) as well as individual and socio-cultural factors (stigma, nonawareness of PMTCT services, lack of spousal and family support, loss to follow-up, negative experiences with hospital staff, the preference for home delivery) have been highlighted as barriers in the literature (Barker et al., 2011; Thielgaard et al., 2011; Chinkonde et.al., 2009; Manzi et al., 2005; WHO, 2005). Nigeria, like most countries in sub-Saharan Africa, is experiencing a high prevalence of HIV with about $4.1 \%$ of the adult population living with the virus (NACA, 2011; UNAIDS, 2008) largely due to heterosexual transmission. Besides heterosexual transmission, vertical transmission of HIV from mother-to-child accounts for more than 90\% of pediatric AIDS cases (NACA, 2011). To reduce the number of mother-to-child HIV infections, the governments of various countries have set a goal of universal access and increased the capacity for the delivery of HIV counseling and testing, prevention of mother-to-child transmission, and provision of ARVs by about 2-, 6-, and 8-fold, respectively (UNAIDS, 2008). Despite this increase, the number of women accessing these services is still low. For instance, after more than 5 years since the goal for universal access was established, less than 7,000 HIV positive pregnant women received ARV prophylaxis, representing about $19 \%$ of the annual targets (Federal HIV/AIDS Prevention and Control Office, 2011).

The purpose of this qualitative study, carried out in select comprehensive sites in Anambra, Nigeria, was to explore the views and perceptions of barriers to PMTCT interventions held by pregnant women and service providers, in order to identify issues that could facilitate or impede access to reducing new pediatric HIV infections. The voices and experiences of these women once extracted would help integrate options in program planning and implementation of MTCT.

\section{Materials and methods}

\section{Study design}

A cross-sectional descriptive method that applied the use of qualitative surveys was used to extract opinions of the clients and service providers in the select sites. The study was carried out in the two tertiary 
university teaching hospitals in Anambra state. These are Nnamdi Azikwe University Teaching Hospital (NAUTH), located in Nnewi and Anambra State University Teaching Hospital (ANSUTH) in Awka due to the presence of comprehensive PMTCT services offered in the two facilities. This facility-based crosssectional study was conducted among pregnant HIV-infected women attending ANC clinics in these two comprehensive hospitals. Women of reproductive age (15-49 years) constitute about $22 \%$ of the total population while $2.4 \%$ of the population is estimated to be pregnant women.

A total of 128 samples were collected. The sample size for this study was determined using an appropriate statistical formula for estimating sample size in health studies.

\section{Data collection}

Qualitative data consisted of focus group discussions and in-depth interviews that were held with clinic attendees, health facility staff, and other key informants. In each facility, four focus group discussion sessions, consisting of 8 or 10 participants were held, equally divided between clinic attendees and health facility staff. Participants were recruited based on homogeneity, convenience, and willingness to participate. Health workers (nurses, HIV counselors, midwives, etc.) in the facilities were recruited if they were affiliated with ANC, labour and delivery, HIV counseling and testing, and PMTCT units. Antenatal clinic attendees were recruited after they were approached, briefed about the purpose of the research and consented to participate in focus groups interviews. In-depth interviews were conducted with one matron/head nurse, one physician, and 2 support group members. All discussions and interviews were conducted in English and where possible the native language, Igbo, was used to throw more emphasis on the theme discussed.

\section{Data analysis}

The qualitative data was analyzed using NVivo version 9 qualitative data analysis Software to extract voice remarks around the themes of the study discussed.

\section{Ethical approval}

Ethical approval and clearance for the study was obtained from the Research Ethics Committees of both comprehensive facilities where large scale PMTCT services are offered. Consent to conduct the study was also obtained from the health facilities. Individual verbal informed consent was obtained from every study participant who agreed to participate in the study based on the study inclusion criteria of having attended ANC clinic more than once and between $18-49$ years. Strict confidentiality and anonymity practices were followed in obtaining voice extracts from the participants.

\section{Results of qualitative study}

\section{Barriers to access and utilization of PMTCT services}

The FGD participants discussed different barriers that prevent proper utilization of ANC/PMTCT services at the health facility. In some of their opinions, health workers are usually unprofessional and seemed incompetent in the way they administer their duties even though they are well educated and trained. At the facility level, the time it takes to see the doctor or test at the lab discourage them from sustaining their access of health care services. Some participants voiced harsh treatment from the service providers. At the family and community levels, stigma and discrimination and poor spousal support were highlighted to be the most recurring reason for poor uptake of PMTCT services in the state. At the individual level, getting off work, financial commitment, being tired of taking the routine ARVs, preference for TBA patronage, ignorance and late presentation to ANC.

In terms of their professionalism and treatment of pregnant mothers in general, some FGD participants voiced their dissatisfaction over the health workers' level of competence and skill in PMTCT service delivery. Speaking her mind, an FGD surmised:

"I do not think I am satisfied with the caliber of some nurses that work at the facility since they do not know how to talk to patients. They are sometimes harsh and refuse to repeat themselves in case some pregnant mothers did not hear what they said earlier. Their skill in handling patients is poor." (25-yearsold pregnant mother).

One of the FGD participants emphasized the need to assign responsible health workers who provide ethically sound health services to mothers. 
Texila International Journal of Public Health

Volume 5, Issue 3, Sep 2017

"They [health workers] are not punctual. They waste the time by talking in a group rather than delivering proper service." (42-year-old pregnant mother)

Speaking on the poor spousal support, the following quote voices the need to sensitize male partners of the need to be more supportive regardless of HIV status:

"My HIV-negative husband was so unsupportive and I had to always sneak out to attend ANC at the facility. He once beat for going to ANC because he felt that people would deduce he was HIV-positive when they see me at the ANC." (25-year-old pregnant FGD).

Most FGDs lacked adequate knowledge of HIV and its potential for MTCT hence late presentation at ANC for HTC. They were unaware of their HIV status or the risk of MTCT of HIV. They got to know about their HIV-positive status after delivery when their young infants started falling sick, which then made the doctor request for their HIV serostatus.

"I did not know about HIV infection and I did not know it is possible to transmit it to my baby. I did not know how important it was for me to book for ANC. If I had known I would have booked my pregnancy early enough at the big hospital." (18-year-old pregnant mother)

"If I had known my HIV status and the risk of MTCT, I would have made myself available for PMTCT, for the sake of my unborn child." (38-year-old mother).

On another theme surrounding where they delivered their babies, most FGDs cited a preference for traditional birth attendants primarily because of the level of care and motherly advice showered on them:

"I prefer delivering my babies at the traditional birth attendant's place. She gives me advice on how to care for my baby and makes me feel at home. She uses old traditional practices passed down from generation on maternal and child care and this usually make me feel I am at the right place. "(37-year old FGD)

"If not for my husband, I would have simply gone to the community traditional birth attendant. She is well experienced than most of the nurses who do not even know how to handle me emotionally during childbirth." (40-year old FGD).

"My mother introduced me to one TBA where I had my last baby because of the complications around the delivery of the previous baby. Since then, I have always wanted to patronize that TBA." (28-year old mother)

Some FGDs stated getting off work as one of the barriers for absolute uptake of PMTCT services. The following quotes stressed on that factor:

"I teach in a private secondary school. The demands of day-to-day school activities makes it difficult for me to attend ANC regularly as the principal would not always grant me permission to leave work." (32-year-old pregnant FGD)

"ANC clinic process takes the whole day. I find it difficult to leave my trade for ANC on a regular basis. If they can hasten the process, it can help me to still come to the hospital and go back to my business." (36-year old FGD)

Most key informants agreed that low staff numbers that provide maternal health services, lack of information and limited community awareness about the ANC/PMTCT services, health care provider's attitude and economic factors were some of the main barriers to maternal health service utilization.

"There is only one federal government-owned public health institution rendering all dimensions of service including PMTCT services and everyone wants to attend because they believe the best personnel are here and services are affordable. So provision of maternal health services including PMTCT to these populations by this facility is very difficult especially with the number of health personnel available." (Service provider)

Most FGD participants explained that the quality of ANC services at private health facilities is better than that at public health centers. They reported that private clinics give priority to clients for immediate intervention and do not keep clients to wait for long time. However, they all agreed that private health facilities are very expensive and their services are unaffordable for most people. Had it not been for the cost of services, most FGD participants preferred the services of private health facilities than public ones.

"I would prefer to go to a private health facility than a public one even if it is expensive. There is a long queuing in public health facilities as the service is free of charge. Private facilities tend and care for you more and give you value for your money than public health facilities. Processes are facilitated at private 
facilities but it is your entire obligation to see through your session, even when you are weak." (A 23-yearold pregnant mother)

In the current study, the service providers identified some critical barriers to the full utilization of PMTCT services in Anambra State, including the difficulty of making decision on the initiation of ARV by HIV-positive pregnant mother due to lack of partner's involvement during HCT. The service providers acknowledged the difficulties of engaging men in HCT services and emphasized the need to involve men if uptake of PMTCT service were to be improved:

"Mothers fear to be tested and this is because they do not know the extent of male partner's reaction to the HIV test result. This is also because there is no way to involve the male partners in the programme. Some mothers change their address and disappear after they learned that they are HIV-positive due to the fear of stigma and discrimination". (Service provider)

The service providers also identified the difficulty of HIV-positive pregnant women to disclose their sero-status to their partners due to fear of divorce because of their economic dependence on men.

"Most women who tested positive for HIV do not disclose their sero-status to their partners due to fear of divorce, stigma or violence. This is something related to female's economic dependence on male partners. Consequently, they [women] are unwilling to take the ARVs at all or take it secretly and incorrectly". (Service provider)

The service providers also mentioned about the problem of PMTCT trained staff turnover and an increased workload among staff resulting in low performance of PMTCT services. This is particularly true for those health care providers working in ANC/PMTCT and labor wards.

"There is a high turnover of people trained on PMTCT, there will be a gap when these people leave their job until new staff are trained to replace them. Some staff members consider PMTCT as an additional service for which they are not very much responsible." (Service provider)

The service providers suggested the following as one of the ways to engage pregnant women for HCT:

"To increase PMTCT service utilization, mother-to-mother support groups should be established in the community. Mother-to-mother support group includes HIV-positive or negative mothers volunteering to teach the community about PMTCT. To trace HIV-positive mother defaulters and exposed infants, nurse health extension workers are assigned in their communities. The group also performs HCT in the community". (Service provider).

In another perspective, some of the service providers voiced too much workload and tight busy schedule on their part. Basically, they pointed out too much service provision and reporting to be done for international development agencies, which indirectly impact on the quality of service provided to pregnant mothers:

"I am the PMTCT focal person and I am involved in so many other duties in the hospital. After ANC visits, I document relevant tools which report my activities in the hospital for upward transmission to donors and various IPs. This is also in addition to conduct some level of care and testing for these pregnant mothers. Included in my work description are some meetings which I have to be in attendance. These also impact on the quality of care assessed by the pregnant mothers that attend ANC here." (Matron)

Another said,

"I have a lot to do in a day. I am so overwhelmed by the activities though I enjoy offering these services because it is my job. I think that we need more nurses to help out in this hospital." (Service provider)

\section{Other views on PMTCT services in the state}

The findings of the study highlighted as obstacles to successful access, utilization and uptake of PMTCT services as discussed by the study participants, were the following: the way health workers treat the mothers (mistreatment), the time taken to get ANC service (long wait time, long wait time to get laboratory tests and obtain results), long walking distance to facility, getting off work, and late presentation to ANC for HIV testing and counselling.

Low utilization of PMTCT programs in the state was also attributed to the fact that male partners showed little or no concern for health issues. This was seen in most of the study participants making decisions for PMTCT services without their partners/spouses. As deduced by the study, the men believed in being in a steady healthy state and needed no services of the hospital in any form. It was also due to a lack of financial commitment to the health of the mother and child during pregnancy by the male partners. 
Mothers also suggested a possibility for reimbursement of transportation fees for ANC attendance. This form of reward is expected to positively reinforce ANC attendances. Other incentives such as provision of insecticide-treated nets, water purification solution (sodium hypochlorite), and the abolishment of all forms of out-of-pocket expenses will further enhance attendance at the ANC clinic. They suggested that the delivery of PMTCT services can be better if staff attitudes were improved as this would encourage more patronage. They opined that the recruitment of more staff would reduce the workload and thus guarantee a better client-staff interaction during ANC visits.

Poor community mobilization was also stressed as one of the limitations for the study participants. In their opinions, the community should be sensitized well enough to accommodate people living with HIV/AIDS. HIV/AIDS is not a death sentence and as such people should change their attitude and behaviour towards people living with the disease. It is just like every other infection which can be treated if detected on time. The need for behaviour communication and change is mandatory in PMTCT HIV programs.

\section{Discussion}

The poor quality of PMTCT services experienced by the HIV women was another barrier identified in this study as the mothers complained of long wait times, judgmental and poor staff attitude. While recruitment of more staff could reduce the burden of work load on health workers, the financial implication may not make this option an easy and immediate solution; therefore, efforts should be geared toward improving the efficiency of the existing workers via training and retraining on issues of PMTCT services. As earlier suggested by Barker et al (2011), the health system efficiency can be optimized by close examination of the clinic flow, documentation of requirements, and other logistical improvements which can maximize the efficiency of services and decrease women's waiting times even with the existing staff strength. Shifting tasks among health workers may be another way of maximizing staff roles where feasible for a smoother service delivery (Zachariah et al., 2009).

Stigma and discrimination was another major barrier identified in this study. This barrier was general as it affected the uptake of PMTCT services at all levels of the cascade. This is in line with studies done by Link \& Phelan, 2001; UNAIDS, 2005; Nguyen et al., 2008. Stigma and discrimination was responsible for the following identified reasons for less than optimized utilization of PMTCT programs in the state.These included why mothers did not come for HIV testing; why mothers did not disclose HIVpositive status to their male partners; why mothers did not want to take ARV medicine in the presence of friends and family members either at home, elsewhere or when they travelled; why mothers did not want to administer NVP to their infants in the presence of family members, in-laws and friends; why mothers did not want to come for ANC visits; why mothers will breastfeed their babies outside their homes but will revert to replacement feeding at home; and partly the reason mothers preferred home delivery by TBAs (Deressa et al., 2014; Anigilaje et al., 2016).

Some of the study participants recommended that government should do more to encourage participation by HIV-seropositive pregnant mothers by creating awareness of the program and instituting free $\mathrm{MNCH}$ programs. Where possible, a reward system should be put in place at the facilities to increase ANC attendance. Also, anti-discrimination acts available at the federal level should be enforced at all levels of government-owned facilities to ensure wide PMTCT acceptance. Mother-to-mother support groups was advocated to be restored by the study participants. This is worthwhile as women tend to connect with those who have experiences in what they are going through and desire to be emotionally nurtured. More strategic efforts need to come in male partner involvement, couple testing and counselling and promoting PMTCT.

\section{Conclusion}

The study highlighted some views, perceptions, experiences, opinions of pregnant mothers accessing care and that of health workers providing care at these facilities. It revealed the fact that PMTCT services are yet not readily accessible, even though they are widely available for all pregnant women especially at the facility level and as such requires innovative strategies which include the involvement of different community members are needed to improve this. To meet UNAIDS plan for eliminating HIV by 2030, public health system strengthening in terms of human capacity, consumables, logistics etc. ought to be a priority all orientated to strategically transform the pace of assessing PMTCT services. There is also an 
urgent need for further implementation research to evaluate and scale-up successful approaches for what works best in different health settings. This research also shows the importance of involving TBAs in some aspects of PMTCT services, and asking women which community members they would like to support them. Without further synergy in this area PMTCT will remain under-utilized and eventually out of reach of HIV-infected pregnant women once it becomes discredited by them with far-reaching implications for future generations.

\section{Recommendations}

The researcher suggests a referral link of traditional birth attendants to health facilities in designing PMTCT HIV programs since quite many of the study participants indicated patronage of TBAs. This will help eliminate chances of spreading the virus to newborns. The study advocates incorporating a system of educating and enlightening TBAs on hygiene, HIV and MTCT of the virus or even involving TBAs in community mobilization for achieving the goals of PMTCT.

\section{References}

[1].Anigilaje, E.A., Ageda, B.R. and Nweke, N.O. (2016). Barriers to uptake of prevention of mother-to-child transmission of HIV services among mothers of vertically infected HIV-seropositive infants in Makurdi, Nigeria. Dove Medical Press, 10:57-72.

[2].Barker, P.M., Mphatswe, W. and Rollins, N. (2011). "Antiretroviral drugs in the cupboard are not enough: the impact of health systems' performance on mother-to-child transmission of HIV," Journal of Acquired Immune Deficiency Syndromes, Vol. 56, No. 2, pp. e45-e48.

[3].Chinkonde, J.R., Sundby, J. and Martinson, F. (2009). "The prevention of mother-to-child HIV transmission programme in Lilongwe, Malawi: why do so many women drop out," Reproductive Health Matters, Vol. 17, No. 33, pp. 143-151.

[4].Dabis, F., Newell, M.L., L. Fransen, L. et al. (2000). "Prevention of mother-to-child transmission of HIV in developing countries: recommendations for practice," Health Policy and Planning, vol. 15, no. 1, pp. 34-42.

[5].Deressa, W., Seme, A., Asefa, A., Teshome, G. and Enqusellassie, F. (2014). Utilization of PMTCT services and associated factors among pregnant women attending antenatal clinics in Addis Ababa, Ethiopia. BMC Pregnancy and Childbirth Vol. 14 p.328-341.

[6].Ezeanolue, E.E., Obiefune, M.C., Yang, W., Obaro, S.K., Ezeanolue, C.O., Ogedegbe, G.G. (2013). Comparative effectiveness of congregation-versus clinic-based approach to prevention of mother-to-child HIV transmission: study protocol for a cluster randomized controlled trial. Implement Sci. 8(1):62.

[7].Federal HIV/AIDS Prevention and Control Office (2011). In Multi-sectoral HIV/AIDS Response Annual Monitoring and Evaluation Report 202 EFY.

[8].Federal Ministry of Health. (2003). National HIV/AIDS and Reproductive Health Survey (NARHS).

[9].Link B. and Phelan, J. (2001). On stigma and its public health implications. Paper presented at: Stigma and Global Health: Developing a Research Agenda; Bethesda, MD. Washington: National Institutes of Health.

[10]. Manzi, M., Zachariah, R., Teck, R. etal. (2005). "High acceptability of voluntary counselling and HIV-testing but unacceptable loss to follow up in a prevention of mother-to-child HIV transmission programme in rural Malawi: scaling-up requires a different way of acting," Tropical Medicine and International Health, Vol. 10, No. 12, pp. 12421250.

[11]. NACA (2012). National Agency for the Control of AIDS; Nigeria Global AIDS Response, Country Progress Report. Nigeria: Abuja.

[12]. National Agency for the Control of AIDS (2011). Factsheet 2011: Update on the HIV/AIDS Epidemic and Response in Nigeria.

[13]. Nguyen, T.A., Oosterhoff, P., Ngoc, Y.P., Wright, P. and Hardon, A. (2008). Barriers to access prevention of mother-to-child transmission for HIV-positive women in a well-resourced setting in Vietnam. AIDS Res Ther 17:57.

[14]. Nkwo, P. (2013). Prevention of mother to child transmission of Human Immunodeficiency Virus: The Nigerian perspective. Annals of Medical and Health sciences Research. 2(1):56-65.

[15]. Painter, T.M., Diaby, K.L., Matia, D.M., etal. (2004). "Women's reasons for not participating in follow up visits before starting short course antiretroviral prophylaxis for prevention of mother to child transmission of HIV: qualitative interview study,” British Medical Journal, Vol. 329, No. 7465, pp. 543-546. 
Texila International Journal of Public Health

Volume 5, Issue 3, Sep 2017

[16]. Siegfried, N., van der Merwe, L., Brocklehurst, P. and Sint, T.T. (2011). "Antiretrovirals for reducing the risk of mother-to-child transmission of HIV infection," Cochrane Database of Systematic Reviews, no. 7, p. CD003510. [17]. Theilgaard, Z.P., Katzenstein, T.L., Chiduo, M.G. et al. (2011). "Addressing the fear and consequences of stigmatization - a necessary step towards making HAART accessible to women in Tanzania: a qualitative study," AIDS Research and Therapy, Vol. 8, No. 1, p. 28.

[18]. UNAIDS, (2013). Progress report on the Global Plan towards the elimination of new HIV infections among children by 2015 and keeping their mothers alive.

[19]. UNAIDS (2011). UBRAF 2012-2015 Country Case Study: Nigeria. Geneva.

[20]. United Nations AIDS Program (2010). http://www.unaids.org/globalreport/Global_report.htm

[21]. United Nations AIDS Program (2008). Epidemiological Fact Sheet on HIV and AIDS: Core Data on Epidemiology and Response. Ethiopia.

[22]. UNAIDS (2005). HIV-related stigma, discrimination and human rights violations: case studies of successful programs.

[23]. World Health Organization (2005). The World Health Report, Geneva, Switzerland.

[24]. World Health Organization (2006). Antiretroviral Drugs for treating Pregnant women and preventing infection in Infants: Towards Universal Access: Recommendations for a Public Health Approach, WHO, Geneva, Switzerland. [25]. World Health Organization (2007). Prevention of Mother to Child Transmission (PMTCT). Briefing note, Department of HIV/AIDS.

[26]. World Health Organization (2010). New guidance on prevention of mother-to-child transmission of HIV and infant feeding in the context of HIV.

[27]. World Health Organization (2010). "Towards Universal Access Progress Report.

[28]. Zachariah, R., Ford, N., Philips, M., etal. (2009). Task shifting in HIV/AIDS: Opportunities, challenges and proposed actions for sub-Saharan Africa. Trans R Soc Trop Med Hyg.103:549-558. 